\title{
Necrotizing fasciitis at a distance in a drug user
}

\author{
Abstract \\ A 24 years old man, known intravenous drug user (IVDU), came to the Emergency \\ Department of a hospital in London complaining of pain and swollen on his left lower \\ leg, refiering a history of an episodious of heroin inyection on his left groin five days \\ previously. \\ On examination he was only presenting clinical feitures of generalized cellulitis in the \\ lower leg, with no areas of induration or localized abscesses. With the diagnosis of \\ cellulitis he was admitted in the hospital to have intravenous antibiotics. \\ Later on, the patient developed an extensive area of necrosis affecting the skin and \\ soft tissues of the lateral aspect of his lower leg with not involvement of the thight, \\ needing a serie of repetitive debridaments of the skin and muscles and broad-spectrum \\ antibiotics, to safe the leg and his life.
}

Following surgical and antibiotic treatment, the patient was transfered to a Plastic Surgical Unit for skin grafting.

This is a rare case of presentation of a severe infection in a drug user that appeared without sign of continuity and at a distance of the site of injection and without an associated septic shock.

Keywords: necrotizing fasciitis, drug abusers
Volume 10 Issue 3 - 2018

\author{
Lucia Heras-Garcia, Jose A Rico-Pecero \\ Department of Orthopaedic and Trauma Surgery, Newham \\ University Hospital, London, England
}

Correspondence: Lucia Heras-Garcia, Chairman of the Hand Unit, Khalifa City A, Sector 38, Compound 20,Villa 6, Abu Dhabi PO Box 42683, Tel +971566191616, Email l.herasg@yahoo.com

Received: March 18, 2018| Published: June 05, 2018

\section{Case}

We present a case of a twenty four years old homeless man, with a history of started taking non-parenteral drugs when he was twelve year of age, and five years previously to this episodious changed to intravenous heroin and crack. He was not in a methadone program and except for Hepatitis $\mathrm{C}$ positive diagnosted four years previously and occasionally alcohol abuse, his medical history was unremarkable.

After he was arrested by the police and during the examination taken by the doctor in the police station he was found to have a swollen lower leg and for that reason he was transfered to the Emergency Department at a near hospital in London.

He refered a history of having pain and redness on his lower leg for the last two to three days, worsening every day since and without a history of trauma or local marks of injection. He remembered that five days previous to that, he inyected heroin and crack on the left groin via an already used $16 \mathrm{G}$ needle, reused four times before.

At that time the patient was admited under the care of the medical team with a diagnosis of cellulitis, presenting a swollen, red and painful left lower leg, more on the postero-lateral aspect, extending to the foot and medial side. On examination the peripheral pulses were present, no areas of induration or localized abscesses were found. The temperature was $38^{\circ} \mathrm{C}$, his blood pressure was $140 / 82 \mathrm{~mm} \mathrm{Hg}$, respiratory rate $16 / \mathrm{min}$, heart rate 105 beats/min and saturation of oxigen $98 \%$ on air.

In general his condition was stable, only a bit febrile, but without sign of septic shock. The routine blood test showed: WBC: 28,6 ,Hb: 13,1 $\mu 1$, Platelets: 336, INR>10, D-Dimer:1.8.
Keeping in mind the diagnosis of cellulitis, the DVT protocol was followed, an Ultrasound Scan of his left leg was organized which showned not evidence of deep venous thrombosis.

After 48 hours of started his treatment with Benzylpenicillin and Flucoxacillin intravenously an area of fluctuation appear on the lateral aspect of the lower leg with discharge on minimal pressure throw a two to three mm opening wound which appears expontaniusly, at that moment the patient was refered to the orthopaedic team for further managment.

A swab was taken for culture and sensitivities and a few hours later and after discussion with a mycrobiologist, Clyndamicin and Gentamicin were added intravenously to his treatment. At this stage, the temperature was under control, and the patient was schreduled for surgery, to have an incision and drainage the following day.

On surgery, it was found a more deteriorated lower leg with a large area of severe necrosis not only affecting the skin, also the subcutaneous fat and associated to a massive involvement of the muscles, on view of this it was performed a extensive resection of the skin, fascias and muscles of the postero-lateral aspect of the leg, and a plan was made for a second debridement after 24 hours (Figure 1). A sample of the skin was sent to mycrobiology for culture and sensitivities.

After the surgical debridement, the clinical condition improved, with reduction of pain, inflamation and even temperature. On the second time that the patient was taking to the operative room, 24 hours after the previous one, it was found that despite the clinical situation, the necrostic area was already extended and at that time it was necessary to resect three more centimetres of skin, fascia and 
muscle which were showing sign of clear necrosis. At the end of the intervention the muscles were re-checked for contraction and fresh bleeding and new swabs, were again taken for culture and sensitivities (Figure 2A) (Figure 2B).

While still waiting for mycrobiology to get the culture results a third review was planned on the following 24 to 48 hours. At this stage, it was surprised to find that the patient was clinically confortable with minimal symptoms despite the agressivity showed by the infection.

In the third debridement, the wound appeared to be clear of infection, with minimal inflamatory reaction and no sings of necrosis.

The microbiology studies showed infection by multiorganisms. Others swabs results never came back.

The Plastic Surgical Unit was contacted, the case was discussed and reviewed and due to the fact that the stage of the tissue was clean and that the infection seems to be controlled the agreement was to have the patient transfered for skin grafting.

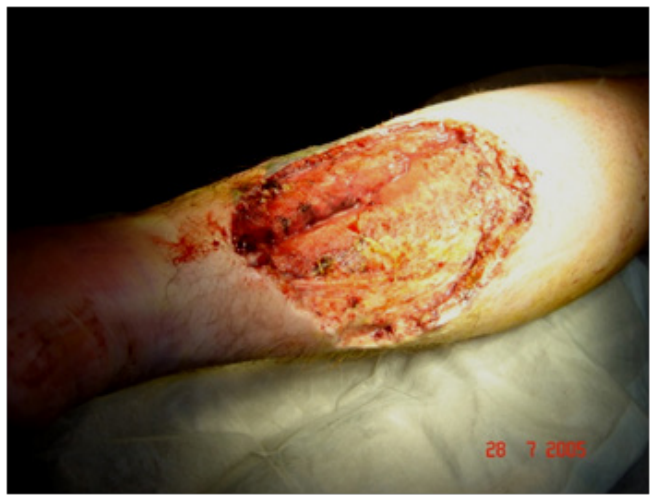

Figure I First debridament after 72 hour of IV antibiotic.

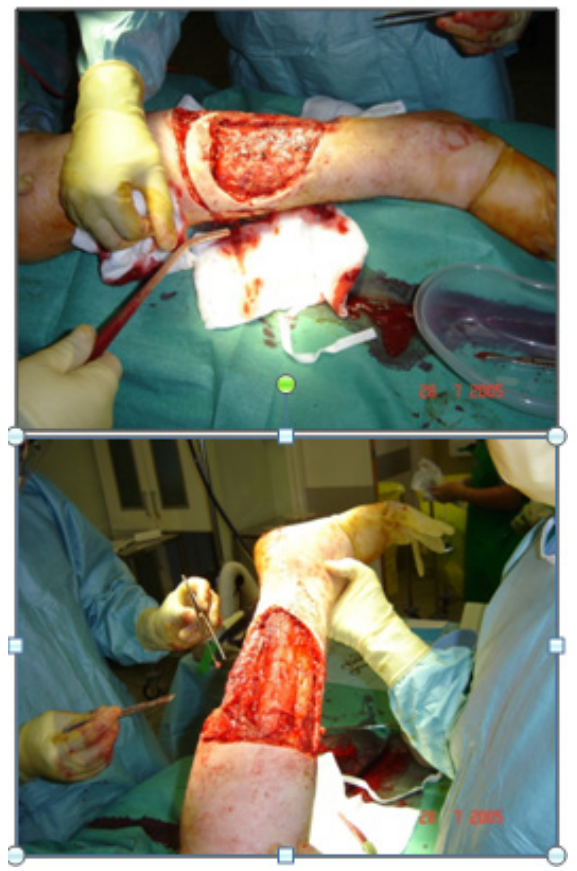

Figure 2(A \& B) In the second look patient needed a new resection of the necrotic tissues.

\section{Discussion}

A series of cases of severe tissues necrosis associated to septic shock in drug abusers was reported in United Kingdon and Republic of Ireland in $2000 .^{1,2}$ The hallmark was severe soft tissue inflamation of the point of drug inyection with spreading necrosis and septic shock with systolic blood pressure below $90 \mathrm{~mm} \mathrm{Hg}$. Most of the patients died in those series. Only few cases has been reported in others countries like Canada and Germany with similar hallmark. ${ }^{3,4}$

We report the first case of Necrotizing Fasciitis in intravenous drug users which did not appeared around the area of the injection but rather at a considerable distance, having the knee joint in between which was not affected by the infection.

This case should make all of us to be aware of the possibility of areas of necrotizing fasciitis in inmunological depresed drug addicts appearing not locally but at a considerable distance, this situation could be explained by the circunstancy that the deprived inmunological response those drug addicts users have may be possible the main reason why an infected trombus will travel all the way distally and settle in areas where the circulation is more deteriorated or more prompt to oedema. We need to be aware of those cases and not only think in local abscess in those individuous.

Recognize the problem and start a fast and aggressive surgical treatment with an extensive debridement, that have to be planned to repeat 24 hours later associated with a broad spectrum of different antibiotic will prevent the septic shock as the subsecuent fatal outcome that we see verey ofthe in drug users.

\section{Acknowledgements}

None.

\section{Conflict of interest}

Author declares there is no conflicts in publishing the article

\section{References}

1. CDC. Unexplained illness and death among injecting-drug usersGlasgow, Scotland, Dublin, Ireland and England, April-June 2000 MMWR Morb Mortal Wkly Rep. 2000;49(22):489-492.

2. Eastern Regional Health Authority and National Disease Surveillance Centre: Serious un-explained illness among injecting drug users in Scotland: Update. Eurosurveillence Weekly. 2000;4:000601.

3. Williamson N, Archibald C, Van Vliet JS. Unexplained deaths among injection drug users: a case of probable Clostridium myonecrosis. CMAJ. 2001;165(5):609-11.

4. Hengge UR, Beiderlinden M, Otterbach F, et al. 31-year-old injection drug user with massive skin necrosis and shock. Dermatology. 2003;206(2):169-71. 